\title{
Ion selective electrodes based on Chalcogenide Glasses
}

\author{
Juan Manuel Conde Garrido ${ }^{\mathrm{a}, 1}$, María Andrea Ureña ${ }^{\mathrm{a}, 2}$ and Bibiana Arcondo ${ }^{\mathrm{a}, 3}$ \\ a Laboratorio de Sólidos Amorfos, Facultad de Ingeniería, INTECIN (Universidad de \\ Buenos Aires - CONICET) \\ Paseo Colón 850, Buenos Aires, Argentina. \\ ${ }^{1}$ imcondegarrido@fi.uba.ar, ${ }^{2}$ murena@fi.uba.ar, ${ }^{3}$ barcond@fi.uba.ar
}

\begin{abstract}
The properties of chalcogenide glasses as sensitive membranes in ion selective electrodes (ISE's) have been investigated. It is shown that ISE's based on the AgGeSe system show sensitivity to the presence of $\mathrm{Ag}^{+}$and $\mathrm{Cu}^{2+}$ ions in aqueous solutions and in both cases they exhibit super-nernstian responses.
\end{abstract}

The analytical properties such as reproducibility, linear range, sensitivity and detection limit were studied.

The response of the electrodes is apparently conditioned by the amount of Ag in the membrane. The addition of $\mathrm{Cu}$ to the membrane composition does not improve the sensitivity of the electrodes.

The sensitivity to $\mathrm{Cu}^{2+}$ ions of thin film ISE's of composition $(\mathrm{AgCu}) \mathrm{GeSe}$ was found to be smaller than the one of bulk ISE's, but still super-nernstian.

\section{Keywords}

Amorphous materials; Ionic conduction; Liquid quenching

\section{Introduction}

Chalcogenide glasses (ChG's) are semiconductor materials with several important properties that make them interesting for their technological applications [1]. One of these applications is the use of ChG's as membranes in ion selective electrodes (ISE's) for the detection of heavy metals in aqueous solutions $\left(\mathrm{Ag}^{+}, \mathrm{Cu}^{2+}, \mathrm{Cd}^{2+}, \mathrm{Pb}^{2+}, \mathrm{Hg}^{2+}\right.$, etc) [2-6].

ISE's based on chalcogenide glasses have many advantages over those commercially available, based on crystalline materials. Some of these advantages include their ability to be built as allsolid-state electrodes (without liquid inner reference), their greater sensitivity, more stable potential, higher selectivity and excellent chemical durability in acidic media due to their high chemical stability [7]. 
In the present, micro-fabrication of sensors and multi-sensor arrays and their use in integrated circuits by means of microelectronics technology are the main objective of research in the chemical sensors field. By these methods different transducting devices can be fabricated, such as $\mu$ ISE, ISFET (Ion Sensitive Field Effect Transistor) and LAPs (Light-Addressable Potentiometric Sensor) [8-10]. An important step in the fabrication of these devices is the deposition of a thin film of sensing material. ChG's turn out to be ideal for this instance since they can be easily deposited as thin films by several techniques, such as RF sputtering, chemical deposition from vapor and pulsed laser deposition (PLD). PLD is one of the simplest, quickest and more versatile techniques and allows the deposition of thin films with complex stoichiometry $[11,12]$.

When ChG's are doped with Ag above a certain threshold concentration, they change from semiconductors to fast ionic conductors and enhance their conductivity from $10^{-13}$ to $10^{-5}-10^{-3}$ S.cm ${ }^{-1}[13]$.

The aim of this work is to investigate the sensing properties of the ChG system AgGeSe as membranes of ion selective electrodes in the presence of $\mathrm{Ag}^{+}$and $\mathrm{Cu}^{2+}$ ions. The basic analytical characteristics of $\mathrm{AgGeSe}$ and $(\mathrm{AgCu}) \mathrm{GeSe}$ systems are reported.

\section{Experimental}

The chalcogenide bulk glasses were prepared from high-purity (4N) elements in compositions: $\left(\mathrm{Ge}_{0.25} \mathrm{Se}_{0.75}\right)_{100-\mathrm{x}} \mathrm{Ag}_{\mathrm{x}}$ with x=10, 15, 20 and 25 at.\% (samples $\left.\boldsymbol{A g x}\right)$ and $\left(\mathrm{Ge}_{0.25} \mathrm{Se}_{0.75}\right)_{75}\left(\mathrm{Ag}_{1-}\right.$ $\left.{ }_{\mathrm{y}} \mathrm{Cu}_{\mathrm{y}}\right)_{25}$ with y=0.05, 0.10, 0.20 at. (samples $\boldsymbol{C u y}$ ) and were synthesized by conventional meltquenching technique as described in Ref. [14].

$\mathrm{X}$-ray diffractometry and scanning electron microscopy (SEM) techniques were used to determine the glassy nature and the uniformity of the glasses.

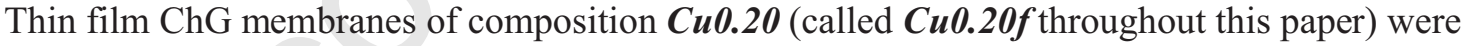
deposited by PLD. A Nd:YAG (Spectra-Physics Quanta Ray model Lab-170-10) pulsed laser in an on-axis geometry was used $(\lambda=266 \mathrm{~nm}, 5 \mathrm{~ns}, 10 \mathrm{~Hz})$. A bulk sample of $\boldsymbol{C u \boldsymbol { 0 } . 2 0}$ was used as target.

All solid state ISE's were constructed by cutting the glasses into rectangular plates. The two faces were then polished with alumina water suspension $(0.3 \mu \mathrm{m})$. Chromium metal contact was sputtered on one side as inner reference and a wire was attached to it with silver paint. The inner reference and the electrical contact were insulated from the aqueous media by encapsulating them in epoxy resin.

The potential measurements were taken by direct potentiometry against a double junction $\mathrm{Ag} / \mathrm{AgCl}$ (sat.) reference electrode. The electrochemical cell used was of the following type: 


\section{$\mathrm{Ag}, \mathrm{AgCl}$ (sat.) $\mid \mathrm{KCl}(4 \mathrm{M})\left\|\mathrm{KNO}_{3}(0.1 \mathrm{M})\right\|$ Analyzed solution | ChG membrane $\mid \mathrm{Cr}$}

Potential measurements were taken at room temperature with a high impedance millivoltmeter with $0.1 \mathrm{mV}$ accuracy. $\mathrm{KNO}_{3}(0.1 \mathrm{M})$ was used as supporting electrolyte in order to insure a constant ionic strength. Test solutions in concentrations from $10^{-2}$ to $10^{-5} \mathrm{M}$ were prepared by successive 10-fold dilutions of a $10^{-1} \mathrm{M}$ solution of the appropriate nitrates $\left(\mathrm{AgNO}_{3}\right.$ and $\mathrm{CuNO}_{3}$ ). Calibrations were carried out at constant stirring in the concentration range $10^{-8}-10^{-3}$ $\mathrm{M}$ by addition of known volumes of test solutions to $100 \mathrm{ml}$ of supporting electrolyte. After the addition of test solutions 2 to 5 minutes were allowed to pass before measuring the potential so that it could reach a stable value.

After each calibration the electrodes were washed in distilled water, allowed to dry and then kept in clean boxes until the next calibration.

Due to the well known sensitivity to light of $\mathrm{Ag}^{+}$solutions, potentiometric experiments were performed in darkness.

\section{Results and discussion}

\subsection{Characterization of the membrane materials}

The XRD patterns of both the quenched alloys and deposited films show the structure typical of amorphous alloys.

Scanning electron microscopy studies revealed that the glasses $\left(\mathrm{Ge}_{0.25} \mathrm{Se}_{0.75}\right)_{100-\mathrm{x}} \mathrm{Ag}_{\mathrm{x}}(\mathrm{x}=10,15$, 20 and 25 at.\%) are non-homogeneous. Their morphology depends on the composition and consists on bright Ag rich zones and dark Ag depleted zones that can be attributed to a widespread liquid miscibility gap near the Se rich corner of the equilibrium phase diagram [15].

\subsection{Analytical characteristics of $A g x$ and $C u y$ electrodes}

The response of the electrodes in the presence of $\mathrm{Ag}^{+}$and $\mathrm{Cu}^{2+}$ ions was studied and the basic analytical characteristics of the calibration curves: linear range, detection limit and slope are reported.

\section{Response to $\mathrm{Cu}^{2+}$ ions}

All $\mathbf{A g x}$ electrodes respond similarly to the presence of $\mathrm{Cu}^{2+}$ ions, with detection limits around $3 \times 10^{-7} \mathrm{M}$, super-nernstian slopes between 60 and $70 \mathrm{mV} / \mathrm{pCu}$ and linear ranges $6 \times 10^{-7}-10^{-4} \mathrm{M}$ (Fig. 1). As the silver content in the membrane composition increases, the slope increases, the detection limit is shifted towards lower concentrations and the linear range is shortened.

The response of the $\boldsymbol{C u y}$ bulk electrodes to the presence of $\mathrm{Cu}^{2+}$ ions is characterized by detection limits around $10^{-7} \mathrm{M}$, super-nernstian slopes between 45 and $60 \mathrm{mV} / \mathrm{pCu}$ and linear 
ranges between $10^{-6}$ and $10^{-4} \mathrm{M}$ (Fig. 2). Samples with lower copper concentration, $\boldsymbol{C u 0 . 0 5}$ (23.75 at.\% of $\mathrm{Ag}$ and 1.25 at. $\%$ of $\mathrm{Cu}$ ) and $\mathbf{C u 0 . 1 0}$ (22.5 at.\% of $\mathrm{Ag}$ and 2.5 at.\% of $\mathrm{Cu}$ ), exhibit a behavior similar to that of $\boldsymbol{A g} 25$ electrodes but with lower slope values $(60 \mathrm{mV} / \mathrm{pCu})$, lower detection limits and slightly longer linear ranges. Samples with higher copper concentration, $\boldsymbol{C u 0 . 2 0}$ (20 at.\% of $\mathrm{Ag}$ and 5 at.\% of $\mathrm{Cu}$ ), exhibit a behavior similar to that of Ag10, $\boldsymbol{A g} 15$ and $\boldsymbol{A g} 20$ electrodes but with lower slope values $(45 \mathrm{mV} / \mathrm{pCu})$. The calibration curves of $\boldsymbol{C u} \mathbf{0 . 2 0 f}$ (thin films) are similar to those of bulk $\boldsymbol{C u 0 . 2 0}$ but with lower slopes (35 $\mathrm{mV} / \mathrm{pCu})$.

Therefore, the study of the response of the electrodes to the presence of $\mathrm{Cu}^{2+}$ ions shows that this response is strongly conditioned by the amount of silver present in the membrane composition. In order to investigate the effect of silver in the sensing mechanism a study of the response of the electrodes to the presence of $\mathrm{Ag}^{+}$ions was performed.

\section{Response to $\mathrm{Ag}^{+}$ions}

The calibration curves of $\boldsymbol{A g x}$ electrodes against $\mathrm{Ag}^{+}$ions exhibit an interesting dependence with time. The evolution of the calibration curves can be observed in Figs. 3 and 4.

The electrodes which had been previously calibrated against both $\mathrm{Cu}^{2+}$ and $\mathrm{Ag}^{+}$ions (named c$(\mathrm{Ag}, \mathrm{Cu})$ in Figs. 3 and 4$)$ are characterized by reversible and repetitive calibration curves with super-nernstian slopes between 63 and $67 \mathrm{mV} / \mathrm{pAg}$.

Two electrodes had been previously calibrated against $\mathrm{Cu}^{2+}$ ions (named c-(Cu) in Figs. 3 and 4). When these electrodes are calibrated against $\mathrm{Ag}^{+}$ions for the first time (Fig. 3) a steep increase in the potential is registered in the medium concentration range and then the slope decreases in the high concentration range. From the second calibration on, the electrodes exhibit the same reversible calibration curves as those which have been calibrated many times both in $\mathrm{Cu}^{2+}$ and $\mathrm{Ag}^{+}$solutions, as shown in Fig. 4 .

The "as prepared" electrodes (which had not been previously calibrated or conditioned) initially exhibit the usual irregular behavior (Fig. 3). According to Vlasov $[16,17]$ the reason is that the modified surface layer (MSL) has not been formed yet. During the following calibrations, the exposition of the membrane to $\mathrm{Ag}^{+}$solutions causes the formation of the MSL and therefore the expected reversible calibration curves are obtained (Fig. 4).

In the concentration range $3 \times 10^{-5}-10^{-4} \mathrm{M}$ a reduction of the potential can be observed (Figs. 3 and 4). In order to investigate this phenomenon one of the electrodes with repetitive calibration curves was chosen and its potential was registered as a function of time as the concentration of $\mathrm{Ag}^{+}$in the analyzed solutions was changed. The result of this experiment for an $\mathbf{A g} 25$ electrode is presented in Fig. 5, the rest of the studied compositions present the same response. 
The electrode was left in a $\mathrm{KNO}_{3}(0.1 \mathrm{M})$ solution for 50 minutes. During this time the potential increased until it reached a steady value. The changes in the potential at low concentrations (first observed at $10^{-7} \mathrm{M}$ ) were the usual ones, as measured for other all-solid state ion selective electrodes [18]. When the concentration of the solution was changed to $3 \times 10^{-5} \mathrm{M}$ a different phenomenon was observed. Immediately after the step, the potential started to decrease rapidly until, after 30 minutes, it reached a steady value. The same behavior was observed when the concentration of the analyzed solution was changed to $6 \times 10^{-5} \mathrm{M}$ and to $10^{-4} \mathrm{M}$. For higher concentrations, the behavior was once again the usual one.

Several papers have been published investigating this type of transient response. But to our knowledge in all these cases the transient response was measured in the presence of two kinds of ions (primary and interfering ions) [19-22] whereas in our investigation, the transient signal was obtained in the presence of only the primary ion.

The response of $\boldsymbol{C u y}$ electrodes to the presence of $\mathrm{Ag}^{+}$is similar to the response of $\boldsymbol{A g x}$ electrodes, but with poorer sensitivity and a more notorious potential decrease in the concentration range $3 \times 10^{-5}-10^{-4} \mathrm{M}$.

\section{Conclusions}

$\boldsymbol{A g x}$ and $\boldsymbol{C u y}$ membranes are sensitive to $\mathrm{Ag}^{+}$and $\mathrm{Cu}^{2+}$ ions with super-nernstian slopes. The sensitivity was not improved by the addition of metals to the glass formulation but the detection limits were lowered and the linear ranges were slightly extended.

The observations made in section 3.2 concerning the response to $\mathrm{Cu}^{2+}$ can be summarized by stating that an increase of the copper concentration in the membrane composition only affects the response of the electrode by lowering the slope and that an increase of the silver concentration in the membrane composition (above 20 at.\%) shortens the linear range.

It appears that $\mathrm{Ag}$ in the membranes plays an important role in the sensing mechanism, which results in a significant increase of the slope of the electrode's calibration curve.

These facts suggest that the potential generation comes from multiple simultaneous sources, i.e. ionic exchange plus adsorption/desorption and even surface reactions $[21,23,24]$.

With regard to the results obtained of the potential as a function of time when the concentration of $\mathrm{Ag}^{+}$in the analyzed solutions is changed, further investigations need to be performed to accurately assert the mechanism that causes the observed behavior in the medium concentration range. 


\section{Acknowledgements}

The authors greatly acknowledge the support of ANPCyT, Universidad de Buenos Aires and CONICET from Argentine.

\section{References}

[1] "Semiconducting Chalcogenide Glass III: Applications of chalcogenide glasses",

Semiconductors and semimetals. Vol 80.Ed: R. K. Willardson and E. R. Weber. Elsevier Academic Press, (2004).

[2] Y.G. Vlasov, E.A. Bychkov, Ed.: J.R.D Thomas, Ion-Selective Electrode Reviews, vol. 9, Pergamon Press, Oxford, UK (1987) 5-93.

[3] Y.G. Vlasov, E.A. Bychkov, A.V. Legin, Talanta 6 (1994) 1059-1093.

[4] Y.G. Vlasov, A.V. Legin, E.A. Bychkov, Sens. Actuators B 24 (1995) 309-311.

[5] Y.Vlasov, E.A. Bychkov, AV. Legin, J. of Analytical Chemistry Vol. 52, 11, (1997) 1073 1079.

[6] J.M. Conde Garrido, F. Macoretta, M.A. Ureña and B. Arcondo, J. Non-Cryst. Solids (2009), doi:10.1016/j.jnoncrysol.2008.12.026.

[7] Yu.G. Vlasov, E.A. Bychkov and A.V. Bratov, Analyst, vol. 119, (1994) 449-454.

[8] J.P. Kloock, Y.G. Mourzina, J. Schubert and M.J. Schöning, Sensors 2 (2002) 356-365.

[9] M. Schöning and J.P. Kloock, Electroanalysis 19, (2007) 2029-2038.

[10] J.P. Kloock, L. Moreno, A. Bratov, S. Huachupoma, J. Xu, T. Wagner, T. Yoshinobu, Y. Ermolenko, Y.G. Vlasov, M.J. Schoning, Sens. and Actuators B: Vol. 118, (2006) 149-155. [11] M.J. Schöning, C. Schmidt, J. Schubert, W. Zander, P. Kordos, H. Lüth, A. V. Legin, B. L. Seleznev, Yu. G. Vlasov, Thin film sensors based on chalcogenide glass materials prepared by pulsed laser deposition technique, in: Proceeding of Eurosensors XII, The Hague, The Netherlands, 12-15. September 1999. 
[12] M. Frumar, B. Frumarova, P. Nemec, T. Wagner, J. Jedelsky, M. Hrdlicka; J. of NonCryst. Solids 352 (2006) 544-561.

[13] A. Piarristeguy, J.M. Conde Garrido, M.A. Ureña, M. Fontana, B. Arcondo, J. of NonCryst. Solids 353 (2007) 3314-3317.

[14] M.A. Ureña, M. Fontana, B. Arcondo, M.T. Clavaguera-Mora; J. Non-Cryst. Solids 320 (2003) 151-167.

[15] A. Prince, in: G. Petzow, G. Effenberg (Eds.), Ternary Alloys, VCH, New York (1988) p. 195.

[16] Yu. Vlasov, E. Bychkov, M. Medvedev, Anal Chim. Acta 185 (1986) 137-158.

[17] Yu. Vlasov, E. Bychkov, Sensors and Actuators 12 (1987) 275-283.

[18] Yu.G. Vlasov, E.A. Bychkov and B.L. Seleznev, Sensors and Actuators B 2 (1990) 23-31.

[19] E. Lindner, K. Toth, and E. Pungor, Anal. Chem. 54 (2) (1982), 202-207.

[20] A. Lewenstam, A. Hulanicki, and T. Sokalski, Anal. Chem. 59 (11) (1987), 1539-1544.

[21] T. Berube, R. Buck, E. Lindner, M. Gratzl, E. Pungor, Anal. Chem. 61 (1989) 453-458.

[22] B. Paczosa-Bator, T. Blaz, J. Migdalski, A. Lewenstam, Bioelectrochemistry 71 (2007) 6674.

[23] G. Papeschi, Sens. Actuators B 24-25 (1995) 336-340.

[24] V.S. Vassilev, S.V. Boycheva, Talanta 67 (2005) 20-27. 
Figure 1: Calibration curves of $\boldsymbol{A g x}$ electrodes in the presence of $\mathrm{Cu}^{2+}$ ions.

Figure 2: Calibration curves of $\boldsymbol{C u y}$ electrodes in the presence of $\mathrm{Cu}^{2+}$ ions.

Figure 3: Calibration curves of $\boldsymbol{A g x}$ electrodes in the presence of $\mathrm{Ag}^{+}$ions.

Figure 4: Calibration curves of $\boldsymbol{A g x}$ electrodes in the presence of $\mathrm{Ag}^{+}$ions, following calibrations.

Figure 5: Potential vs. Time of an $\boldsymbol{A g} 25$ electrode as $\mathrm{Ag}^{+}$concentration in the solution was changed. 


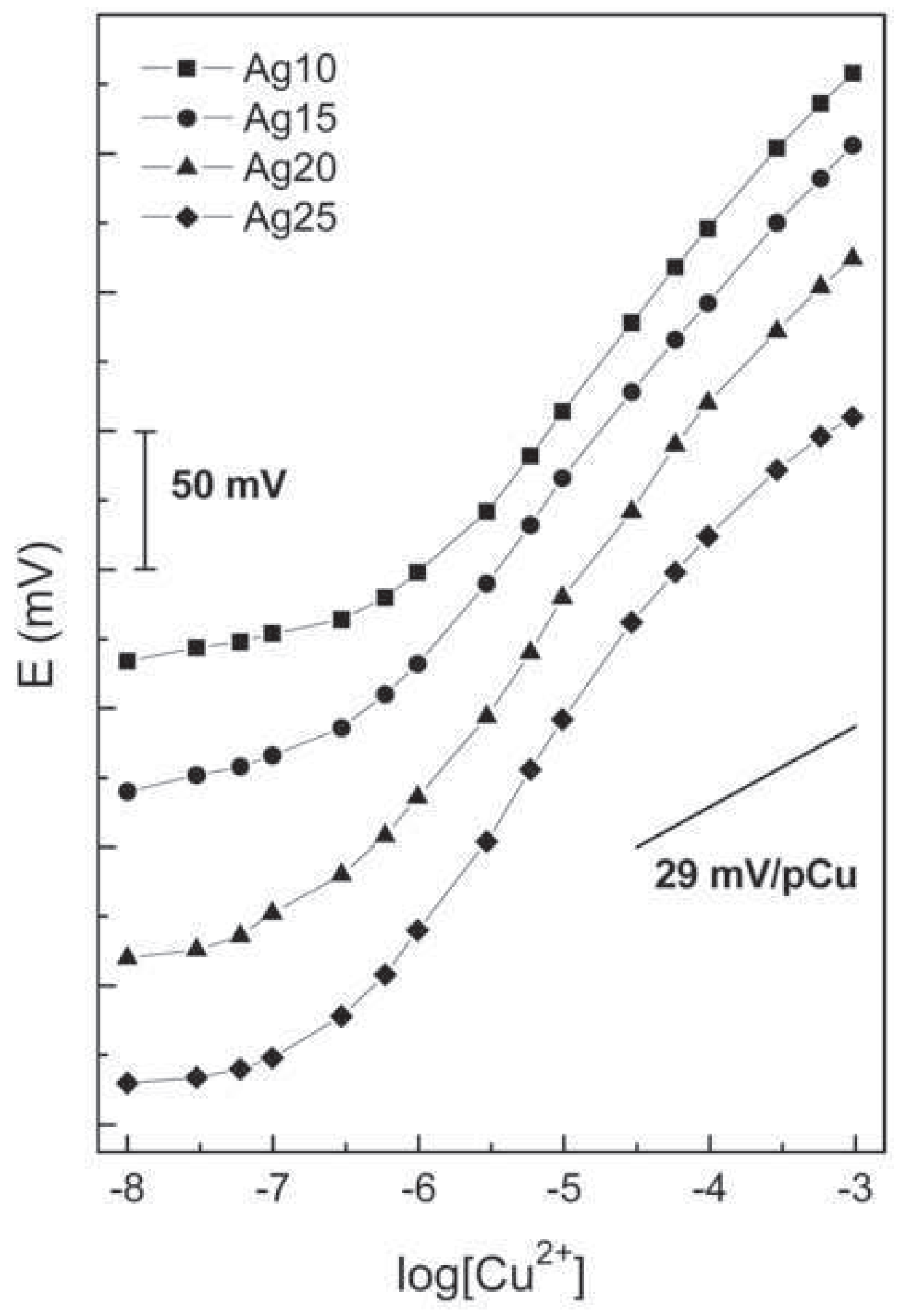




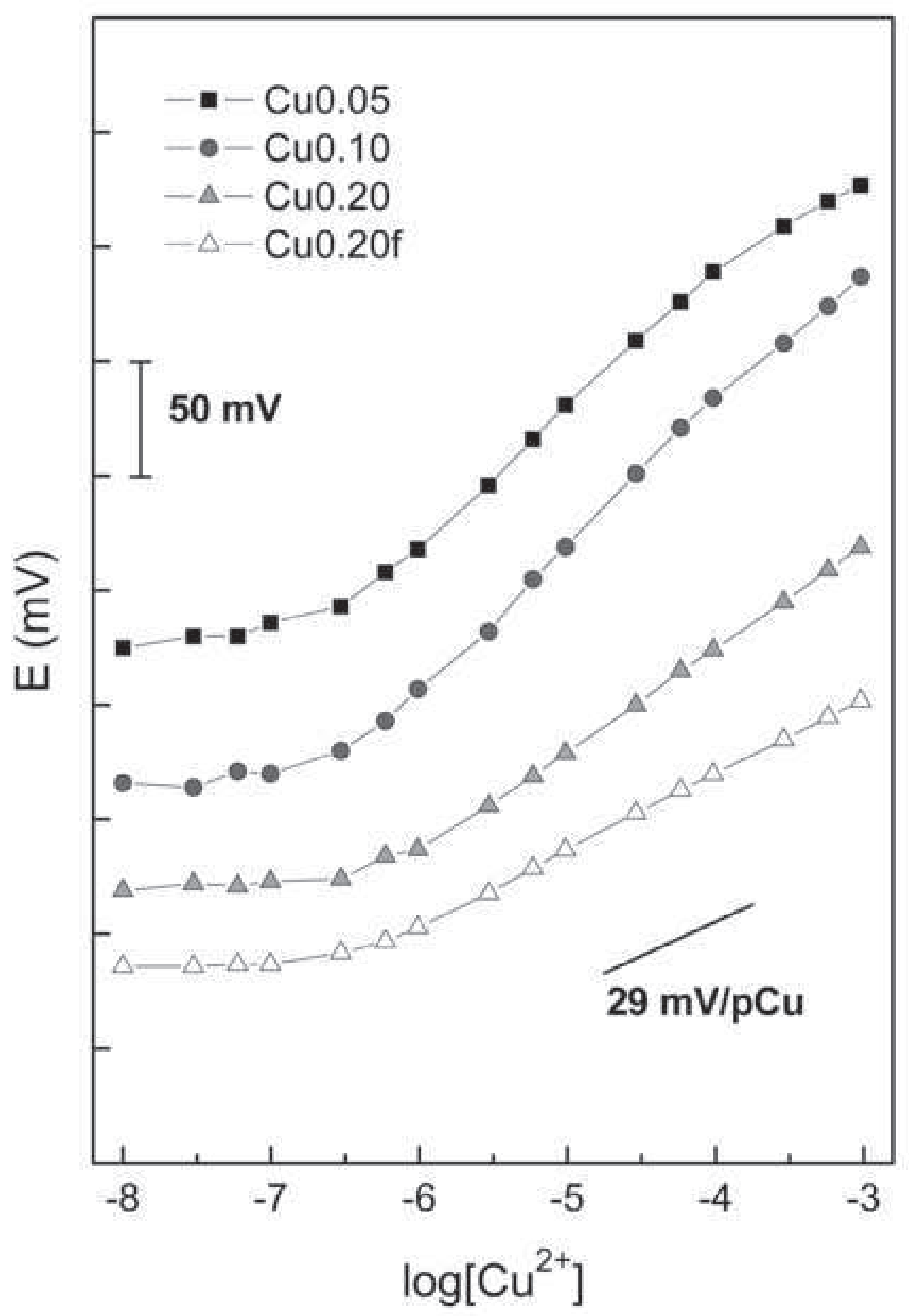




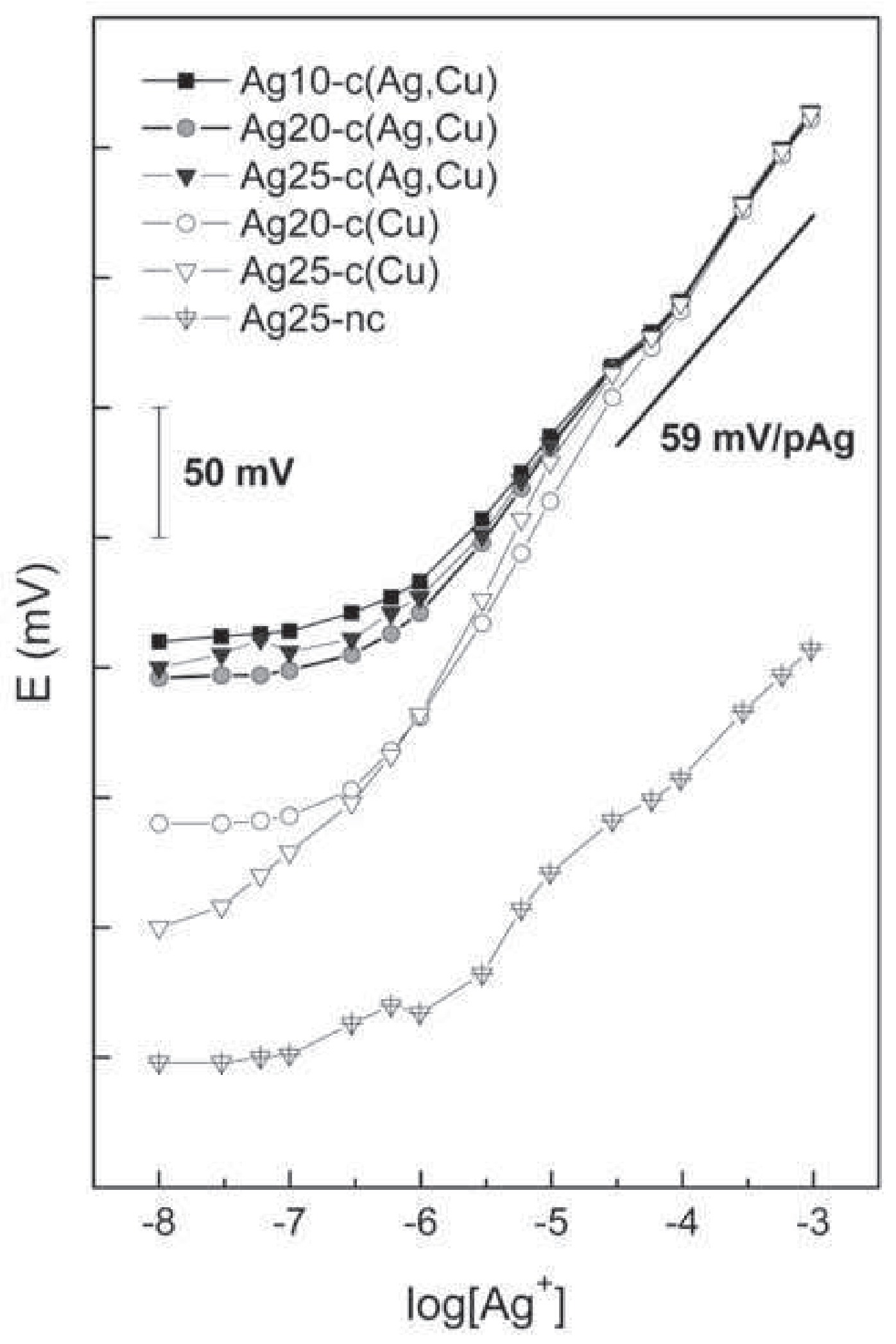




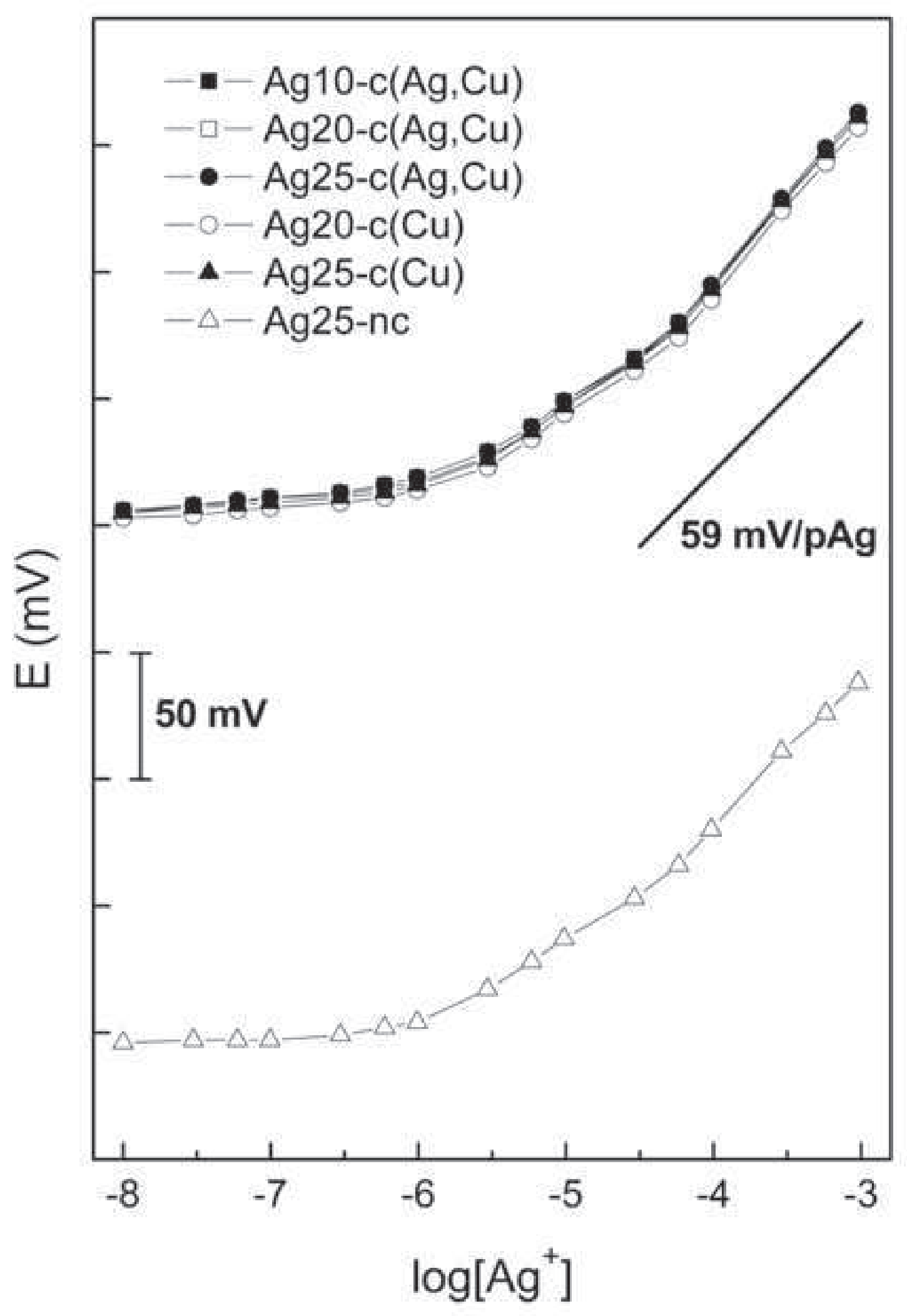




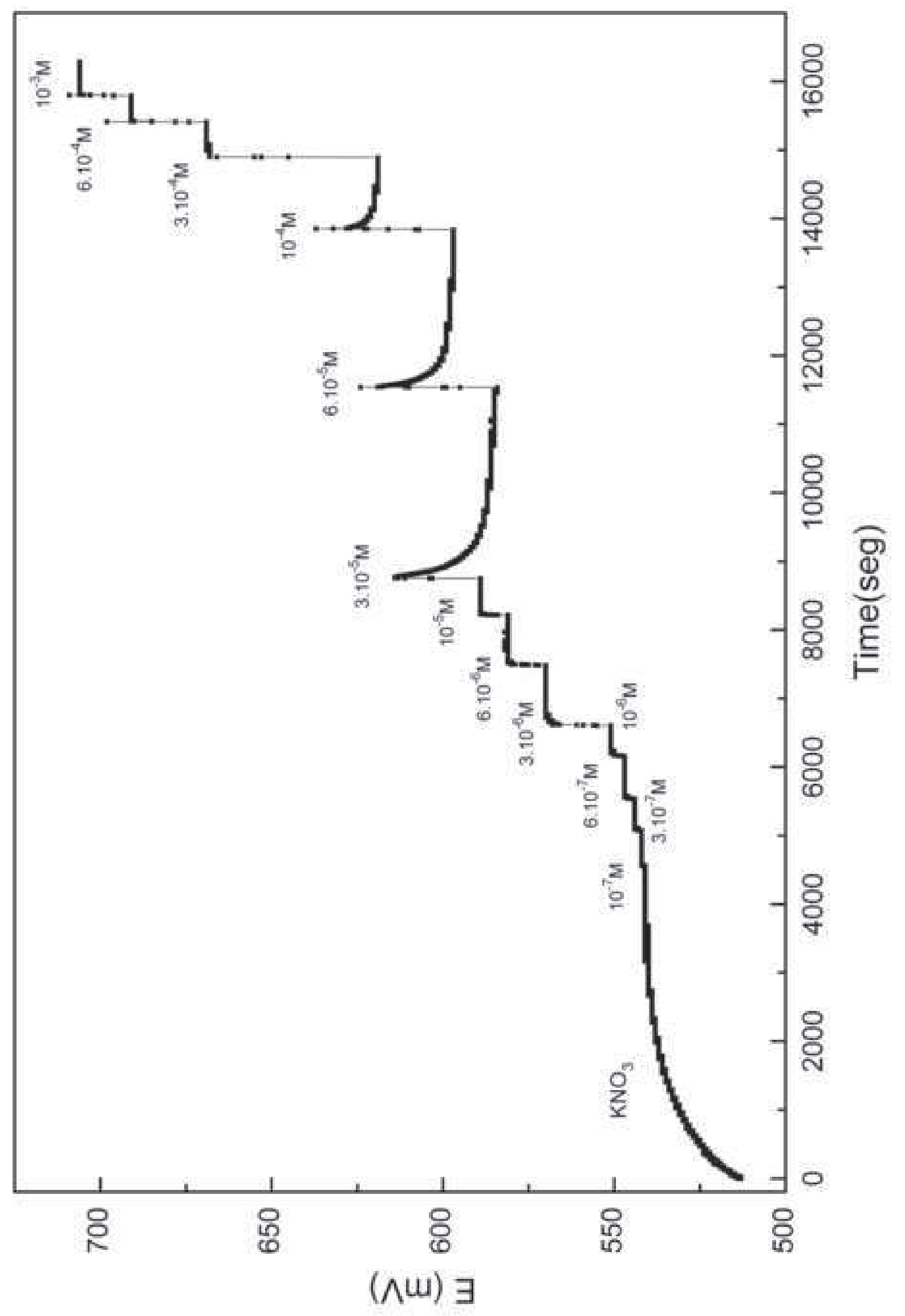

